$0006-2952(94) 00290-8$

\title{
INTESTINAL CLEARANCE OF H2-ANTAGONISTS
}

\author{
Yuk-Fung Hui, Joseph Kolars*, Zhenze Hu and David Fleisher ${ }^{+}$ \\ College of Pharmacy and Department of Medicine, University of Michigan, \\ Ann Arbor, MI 48109, U.S.A. \\ (Accepted 7 June 1994)
}

\begin{abstract}
Jejunal perfusion of cimetidine resulted in the appearance of lumenal cimetidine sulfoxide in both rats and humans. In the rat, ileal perfusion yielded negligible sulfoxide metabolite as compared with that of the jejunum. Jejunal co-perfusion of an anionic-exchange inhibitor, 4,4'-diisothiocyanostilbene-2,2'-disulfonic acid, blocked the appearance of drug sulfoxide, and methionine co-perfusion yielded concentration-dependent inhibition of lumenal cimetidine sulfoxide. Intravenous injection of high concentrations of cimetidine sulfoxide did not produce detectable lumenal metabolite levels during jejunal perfusion of drug-free buffer, providing in silu evidence that lumenal metabolite is generated by the small intestine. The extent of the appearance of lumenal sulfoxide was significantly greater for cimetidine than for the other three marketed $\mathrm{H}_{2}$-antagonists in rat jejunum. Variable intestinal clearance of this extensively prescribed class of therapeutic agents may contribute to their absorption variability.
\end{abstract}

Key words: cimetidine; $\mathrm{H}_{2}$-antagonists; intestinal metabolism; sulfoxide metabolite

The oral administration of $\mathrm{H}_{2}$-receptor antagonists has been of therapeutic utility in the treatment of gastric and duodenal ulcers, as well as gastroesophageal reflux disease. There are currently four $\mathrm{H}_{2}$-antagonist prescription drugs on the U.S. market: cimetidine, ranitidine, famotidine and nizatidine. These drugs are characterized by incomplete and variable absorption, resulting in drug plasma level variability. The incomplete absorption has been attributed to the poor intestinal membrane permeation (1) of these weakly basic drugs, which are ionized at intestinal $\mathrm{pH}$. The coupling of variable small intestinal residence time and limited membrane permeation is responsible for much of the absorption variability (2).

In the course of an in situ rat jejunal perfusion study investigating the effects of mucosal microclimate $\mathrm{pH}$ on cimetidine ionization and absorption (3), a substantial quantity of metabolite was detected by HPLC analysis of the exiting perfusate. Confirmation of metabolite identity and subsequent characterization of $\mathrm{H}_{2}$-antagonist intestinal clearance are the subjects of this report. The metabolite was observed to coelute with standard cimetidine sulfoxide, and identification was later verified by mass spectrometry. Cimetidine jejunal perfusions in two human subjects also yielded the sulfoxide metabolite in the lumenal contents.

Drug sulfoxidation in animal small intestinal microsomal preparations was first documented for phenothiazines in 1977 (4), and intestinal sulfoxidation of albendazole, an anthelmintic drug, has been reported recently (5). While phase II metabolism is more commonly cited as an intestinal clearance mechanism (6), phase I cyclosporin metabolism in rat (7) and human (8) intestine has

*Current address: 3913 Taubman Center, Department of Medicine, University of Michigan, Ann Arbor, MI 48109-0362, U.S.A.

+Corresponding author. Tel. (313) 764-2070; FAX (313) 7632022.

\#Abbreviations: $\quad \mathrm{H}_{2}, \quad$ histamine ${ }_{2}$; DIDS, 4,4'diisothiocyanostilbene-2,2'-disulfonic acid; MES, 2-(Nmorpholino)ethanesulfonic acid; and PEG, polyethylene glycol. been shown to influence oral cyclosporin absorption variability (9).

In addition to a comparison of intestinal sulfoxidation of the commercially available $\mathrm{H}_{2}-$ antagonists, this study provides preliminary information on regional and flow rate dependence of cimetidine sulfoxidation, as well as identification of inhibitors of metabolic clearance.

\section{Materials and Methods}

Materials. Cimetidine, famotidine, ranitidine, DIDS, methionine, sodium and potassium chloride, MES buffer and PEG 4000 were obtained from the Sigma Chemical Co. (St. Louis, MO). Carbon-14 labeled PEG 4000 was obtained from New England Nuclear, Dupont (Wilmington, DE). Nizatidine, cimetidine sulfoxide and famotidine sulfoxide were gifts from Eli Lilly, SmithKline Beecham and the Yamanouchi Pharmaceutical Co., respectively.

Rat perfusions. Experimental details of the singlepass in situ rat intestinal perfusion technique have been reported previously (1). A midline incision was made in anesthetized rats; inlet Tygon tubing was inserted into the jejunum $10 \mathrm{~cm}$ distal to the ligament of Treitz, and an outlet tube was inserted $6-8 \mathrm{~cm}$ distal to the inlet. In some studies, a second perfusion was carried out in the ileum with the outlet tubing 2 $\mathrm{cm}$ proximal to the ileocecal junction and the inlet tube $6-8 \mathrm{~cm}$ proximal to the outlet. Iso-osmotic perfusion solutions containing $5 \mathrm{mM}$ potassium chloride and $135 \mathrm{mM}$ sodium chloride were adjusted to $\mathrm{pH} 6.5$ using $10 \mathrm{mM}$ MES buffer. Some of the ileal perfusions were performed at $\mathrm{pH} 7.5$ using HEPES buffer. The non-absorbable marker, $0.01 \%$ PEG 4000 traced with [ ${ }^{14}$ C]PEG 4000 (specific activity $3.2 \times 10^{6} \mathrm{dpm} / \mu \mathrm{mol}$ ), was included to correct drug and metabolite concentration for intestinal water transport. Perfusion $\mathrm{H}_{2}$-antagonist concentrations were $0.4 \mathrm{mM}$, consistent with projected jejunal concentrations from a typical human famotidine oral dose $(40 \mathrm{mg}$ ) taken with 8 ounces of fluid $(0.5 \mathrm{mg}$ in a 2-mL gastric fluid load in rat). Cimetidine 
perfusions were also performed at $4 \mathrm{mM}$, consistent with projected jejunal concentrations from a typical cimetidine oral dose $(400 \mathrm{mg})$.

Human perfusions. Cimetidine $(4 \mathrm{mM})$ jejunal perfusions were carried out in two human volunteers. An Andersen perfusion tube (four lumen) was placed via the mouth $60 \mathrm{~cm}$ into the small intestine. The distal tip of the tube was in the jejunum approximately $40 \mathrm{~cm}$ beyond the ligament of Treitz. Correct positioning of the tube was confirmed via periodic fluoroscopy. Once in place, a tube-mounted balloon was inflated to approximately $20 \mathrm{cc}$ to provide gentle occlusion of the duodenal lumen. (This prevents bile metabolites from contaminating the perfusate.) Perfusion of drug solution was from a tubing port just distal to the balloon occluding the duodenum. Jejunal samples were aspirated from a suction port approximately $50 \mathrm{~cm}$ distal to the site of perfusion at time zero and every $10 \mathrm{~min}$ after initiation of perfusion for up to $1 \mathrm{hr}$. Measurements of low trypsin activity were used to document effective occlusion. Trypsin was measured via a titrimetric method using para-tosyl-L-arginine methyl ester as a substrate (10). PEG 4000 was included as a non-absorbable marker so that druy and metabolite concentrations could be corrected for intestinal fluid absorption/secretion. PEG 4000 concentrations were assessed by a turbidimetric measurement (11).

Measurement of drug and metabolite. Perfusate concentrations of drug and metabolite were determined from standard curves made in blank perfusate. Neither drug oxidation nor metabolite reduction was obscrved in standard solutions. Results are reported from a reverse phase assay. An aqueous mobile phase (made at $\mathrm{pH} 3$ with phosphoric acid) containing $3.5 \%$ acetonitrile and $1 \%$ triethylamine was pumped through an RP-18 column at 1.4 $\mathrm{mL} / \mathrm{min}$. Detection of the sulfoxide at $3 \mathrm{~min}$ and drug at $8.5 \mathrm{~min}$ was carried out at $228 \mathrm{~nm}$. Co-elution of standard sulfoxide with metabolite was also confirmed with normal phase and ion-pairing HPLC assays. The metabolite assay limit of detection was $50 \mathrm{nM}$.

Metabolite identification. Rat and human perfusate metabolite was identified initially as the sulfoxide since spiking perfusate samples with standard cimetidine sulfoxide and famotidine sulfoxide showed that the suspected metabolite co-eluted with standard.

MS analyses were carried out on HPLC fractioncollected single rat and human cimetidine perfusate samples and compared with standard cimetidine and standard cimetidine sulfoxide. Analyses were performed utilizing an Autospec Ultima-Q hybrid mass spectrometer (VG Analytical Ltd., Manchester, UK) of EBEqQ geometry. Ionization was by LSIMS, using cesium ions with energies of $20 \mathrm{keV}$ for the primary beam. The liquid matrix was 3-nitrobenzyl alcohol. One microliter of HPLC fraction was applied to the probe. These analyses confirmed the identification of cimetidine sulfoxide as the metabolite HPLC peak from rat and human jejunal cimetidine perfusions.

\section{Results and Discussion}

Comparison of rat jejunal $\mathrm{H}_{2}$-antagonist sulfoxidation. The lumenal appearance of $\mathrm{H}_{2}-$ antagonist sulfoxide metabolite was quantitated as the molar ratio of sulfoxide to parent drug in the exiting perfusate. The results (normalized for the length in centimeters of the perfused intestinal segment) are displayed in Fig. 1. Standard sulfoxide metabolites were only available for cimetidine and famotidine. Peaks eluting at times equivalent to cimetidine and famotidine sulfoxide standards from nizatidine and ranitidine perfusions were treated as sulfoxide metabolite.

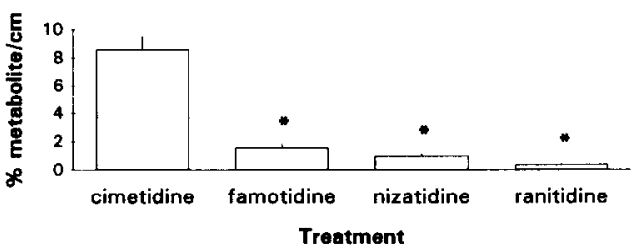

Fig. 1. Mean molar percent sulfoxide metabolite $( \pm$ SEM) for each drug, shown per centimeter of perfused jejunal length. Perfusions were performed at $\mathrm{pH} 6.5$ and a flow rate of $0.12 \mathrm{~mL} / \mathrm{min}$. Inlet drug concentrations were performed at $0.4 \mathrm{mM}$ for each $\mathrm{H}_{2}$-antagonist with at least 4 animals per drug. * Significantly different from cimetidine at the $P=0.05$ level by Student's t-test.

Cimetidine sulfoxide: Dependence on route of administration, intestinal region, perfusion concentration and flow rate. Neither cimetidine nor the sulfoxide metabolite was observed in exiting jejunal perfusate of drug-free buffer following intravenous administration of $1 \mathrm{~mL}$ cimetidine sulfoxide $(4 \mathrm{mM})$. This result suggests that metabolite from drug perfusion is of intestinal rather than systemic origin.

Considerably less sulfoxidation was observed in rat ileum as compared with jejunal cimetidine perfusions (Fig. 2). At a perfusion flow rate of $0.19 \mathrm{~mL} / \mathrm{min}$, the percent of jejunal cimetidine sulfoxidation was not different over a 10-fold concentration range (calculated on the basis of the typical oral dose for this class of agents).

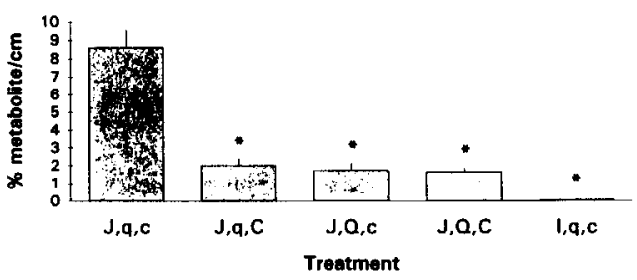

Fig. 2. Percent of cimetidine sulfoxide appearing in exiting perfusate as a function of intestinal region ( $J=$ jejunum, $I=$ ileum), perfusion flow rate $(q=0.12$ $\mathrm{mL} / \mathrm{min}, Q=0.19 \mathrm{~mL} / \mathrm{min}$ ) and drug concentration $(C=4 \mathrm{mM}, c=0.4 \mathrm{mM})$. * Significantly different from $J, q, c$ at the $P=0.05$ level by Student's $t$-test. 
Decreasing the perfusion flow rate to $0.12 \mathrm{~mL} / \mathrm{min}$ significantly increased the percentage of jejunal cimetidine sulfoxidation (Fig. 2). These results suggest that the extent of $\mathrm{H}_{2}$-antagonist sulfoxidation will be a strong function of drug residence time coupled to clearance capacity in upper and lower small intestine. It is projected that variable regional residence times contribute to the drug plasma level double peaks (two drug blood concentration maxima following oral administration) observed in clinical practice.

Human jeiunal cimetidine sulfoxidation. To assess the clinical relevance of these findings, preliminary 4 $\mathrm{mM}$ cimetidine perfusions were carried out in two human subjects at $10 \mathrm{~mL} / \mathrm{min}$ over $50 \mathrm{~cm}$ of jejunum. The perfusate content of cimetidine sulfoxide was $6 \%$ in the first subject and $3 \%$ in the second subject. While this is considerably less sulfoxidation than observed in rat jejunum, slower perfusion flow rates at a lower cimetidine concentration are projected to produce a greater extent of lumenal metabolite.

Inhibitors of cimetidine sulfoxide appearance in the rat jejunal lumen. As these studies were undertaken originally to investigate the effect of mucosal microclimate $\mathrm{pH}$ on variable absorption of cimetidine, amiloride and DIDS were employed as inhibitors of mucosal sodium-proton and chloride-bicarbonate exchange (3). Inclusion of $100 \mu \mathrm{M}$ DIDS in $0.4 \mathrm{mM}$ cimetidine perfusions reduced the levels of an early eluting peak later established as the sulfoxide metabolite (Fig. 3).

Subsequent to identification of cimetidine sulfoxidation, potential inhibitors were selected from products of intestinal sulfur-containing amino acid metabolism. Cimetidine co-perfusion with taurine and replacement of perfusate sodium chloride with equal molar sodium sulfate failed to inhibit cimetidine sulfoxide appearance in the jejunal lumen (data not shown). Co-perfusion of $0.4 \mathrm{mM}$ cimetidine with 2.5 $\mathrm{mM}$ glutathionc reduced the appearance of cimetidine sulfoxide in the rat jejunal lumen. Co-perfusion of methionine produced a concentration-dependent reduction in lumenal cimetidine sulfoxide (Fig. 3).

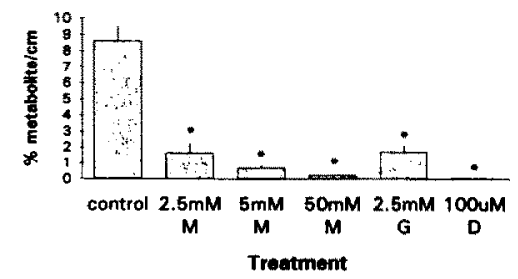

Fig. 3. Percent of cimetidine sulfoxide appearing in rat jejunal lumen in the presence of co-perfused inhibitors ( $D=D I D S, G=$ glutathione, $M=$ methionine). Cimetidine perfusate concentration: $0.4 \mathrm{mM}$; perfusion flow rate: $0.12 \mathrm{~mL} / \mathrm{min}$. *Significantly different from control at the $P=0.05$ level by Student's t-test.

While the DIDS results suggest that metabolite export might be blocked, lumenal methionine sulfoxide was not detected from cimetidine coperfusion with methionine. More mechanistic studies are in progress.
Acknowledgements - The authors would like to thank Dr. Roger Hayes of Parke Davis/Warner Lambert for identifying the sulfoxide metabolite in rat and human perfusate by mass spectrometric analysis and Dr. Paul Watkins of the University of Michigan Clinical Research Center for advice and clinical study support. This work was supported by an Upjohn Research Endowment Award.

\section{REFERENCES}

1. Amidon GL, Sinko PJ and Fleisher D, Estimating human oral fraction dose absorbed: A correlation using rat intestinal membrane permeability for passive and carrier-mediated compounds. Pharm Res 5: 651-654, 1988.

2. Oberle $R L$ and Amidon GL, The influence of variable gastric emptying and intestinal transit rates on the plasma level curve of cimetidine; an explanation for the double peak phenomenon. I Pharmacokinet Biopharm 15: 529-544, 1987

3. Hu $\mathrm{Z}$, Glucose effects on intestinal drug absorption: Contributions of microclimate $\mathrm{pH}$ and the paracellular pathway. Ph.D Thesis, University of Michigan, 1992.

4. Knoll R, Christ W, Muller-Oerlinghausen B and Coper H, Formation of chlorpromazine sulfoxide and monodesmethylchlorpromazine by microsomes of small intestine. Naunyn Schmiedebergs Arch Pharmacol 297: 195. $200,1977$.

5. Lawrenz VA, Eglit $S$ and Kroker $R$, Investigation of the metabolism of albendazole in the isolated perfused intestine of rat. Disch Tierarztl Wochenschr 99: 416-418, 1992.

6. Goon $D$ and Klaassen $C D$, Dose-dependent intestinal glucuronidation and sulfation of acetaminophen in the rat in situ. J Pharmacol Exp Ther 252: 201-207, 1990.

7. Kolars JC, Stetson PL, Rush BD, Ruwart MJ, SchmiedlinRen P, Dueil EA, Voorhees JJ and Watkins PB, Cyclosporin metabolism by P450IIIA in rat enterocytes: Another determinant of oral bioavailability? Transplantation 53: 596$602,1992$.

8. Kolars JC, Merion RM, Awni WM and Watkins PB, Firstpass metabolism of cyclosporin by the gut. Lancet 338 $1448-1490,1991$.

9. Wu CY, Hebert MF and Benet LZ, Use of IV and oral drug levels from cyclosporin with concomitant rifampin to differentiate gut absorption and metabolism. Pharm Res 10: S-345, 1993.

10. Pelot D and Grossman MI, Distribution and fate of pancreatic enzymes in the small intestine. Am J Physiol 202: 285-288, 1962

11. Hyden $\mathrm{S}, \mathrm{A}$ furbidimetric method for the determination of higher polyethylene glycols in biological materials. $K$ Lantbrukshogsk Ann 22: 1139-1145, 1956. 